\title{
An Enhanced Operational Definition of Dielectric Breakdown for DC Voltage Step-up Tests
}

\author{
Allen Andersen and JR Dennison \\ Materials Physics Group \\ Utah State University \\ 4415 Old Main Hill, Logan, UT 84322 USA \\ allen.andersen@aggiemail.usu.edu, jr.dennison@usu.edu
}

\begin{abstract}
The imprecise definition of breakdown in the ASTM D3755-14 standard can misidentify breakdown. If the recommended test circuit current sensing element threshold is set too high, breakdown may occur undetected. Conversely, false positives may result from designating a low current threshold. An operational definition of breakdown much less sensitive to these pitfalls is outlined herein. This enhanced definition of breakdown is based on the average rate of change of the leakage current with increasing voltage, rather than a simple current threshold, avoiding ambiguous association with anomalies in current traces. For tests that continuously monitor leakage current, breakdown can be detected by a transition from negligible current to an ohmic slope defined by the circuit's current limiting resistors. In practice, a fixed current threshold is inadequate to define dielectric breakdown. Field-enhanced conductivity, partial discharge, surface flashovers, incomplete breakdowns, and other phenomena may further obscure the characteristic dielectric breakdown signature. Pre-breakdown anomalies in current traces can also now be clearly identified and studied, in addition to the breakdown itself.
\end{abstract}

\section{INTRODUCTION}

Many dielectric breakdown test systems rely primarily on leakage current measurements to identify breakdown, particularly those following the ASTM D3755-14 Standard Test Method for Dielectric Breakdown Voltage and Dielectric Strength of Solid Electrical Insulating Materials Under DirectVoltage Stress [1]. Such measurements are critical for applications including high voltage direct current (HVDC) power, high voltage switching and control, microelectronics, spacecraft charging induced electrostatic discharge (ESD) mitigation, and advancing the understanding of breakdown physics in disordered dielectrics [2-4]. NASA handbooks recommend using the ASTM D3755 test method for dielectric breakdown testing of spacecraft dielectrics [5-7]. A discussion on test methods and their interpretation from a materials physics perspective is found in [3].

This paper discusses challenges associated with accurately identifying breakdown using this measurement technique and practical considerations for improving the interpretation of observed phenomena. We propose an enhanced breakdown criterion that mitigates the difficulties we have experienced with measurements of this type. We identify breakdown based on the average time rate of change of leakage current as opposed to simply a current threshold. DC breakdown tests are emphasized here; however, the results may be easily adapted to AC breakdown testing.

\section{ISSUES WITH STANDARD BREAKDOWN CRITERIA}

In this section we review selected statements from ASTM D3755-14 section 8 Criteria of Breakdown [1]:

"8.1 Dielectric breakdown is generally accompanied by an increase in current in the test circuit that will activate a sensing element such as a circuit breaker, a fuse, or currentsensing circuit. If sensitivity of the element is well coordinated with the characteristics of the test equipment and the material under test, its operation will be a positive indication of breakdown.

8.2 Failure of a circuit breaker to operate is not be a positive criterion of the absence of breakdown. A breaker can fail to trip because it is set for too great a current or because of malfunction. On the other hand, if the tripping circuit is set for too low a current, currents due to leakage or partial discharge (corona) will cause it to trip before breakdown voltage is reached.

8.3 Observe the specimen during the test to ascertain that tripping of the breaker or current-sensing circuit is not caused by flashover...

8.4 Observation of actual rupture or decomposition is positive evidence of specimen breakdown. In test position, however, these physical evidences of breakdown are not always apparent. If breakdown is in question, it is common practice to repeat the test on the same specimen. Breakdown is confirmed when reapplication of test voltage results in a substantially lower breakdown voltage.”

This breakdown criterion defines breakdown as a sudden increase in observed current above some threshold value implemented in analog hardware. As the true breakdown threshold is not easy to predict a priori, the ASTM standard cautions that a threshold set too low may be sensitive to subbreakdown current such as partial discharge (PD). On the other hand, if the threshold is set too high, breakdown may occur without reaching the arbitrary current threshold. While postbreakdown observation of visual damage or repetition of the test on a particular sample can sometimes clarify a questionable or missed breakdown event, information about the breakdown voltage or time is often lost. Because voltage stress history can affect the breakdown potential, previous stress applied to an unbroken sample in an initial test attempt may invalidate subsequent test attempts [2]. 
The USU Material Physics Group (MPG) ESD system continuously monitors the leakage current during HVDC breakdown tests [2]. Even at breakdown, observed currents in this system are generally well below $50 \mu \mathrm{A}$, precluding the use of even the smallest commonly available fuses rated down to $\sim 2 \mathrm{~mA}$ [8]. The equivalent of a 'fuse' in MPG experiments would be some threshold current value that, if reached, defines breakdown. This criterion alone is insufficient, since as shown in Fig. 1, observed pre-breakdown current can-at least temporarily-be a large fraction of the nominal breakdown current set by the current limiting resistors $\left(R_{l m}\right)$ in the circuit, shown by a black dashed line. Observed pre-breakdown current traces include PD and field-enhanced conductivity. Blue dashed lines in Fig. 1 show arbitrary current thresholds that would miss the breakdown voltage (A), a threshold that would correctly identify the breakdown voltage (B), and two thresholds (C and D) where PD would have tripped the current sensing element early.

Due to the continuous monitoring of leakage current, in most cases as in Fig. 1, breakdown is easily visible as a transition from very low current or intermittent PD to a large current, set by the current-limiting resistors, $R_{l m}$. This is especially true at slow voltage ramp rates. We typically recorded the breakdown voltage as the average of the two voltages on either side of this transition. This method of identifying breakdown was severely challenged in cases without a clear transition to a smooth ohmic slope, such as those shown in Fig. 2.

Fig. 2 (a) shows two cases that lack a clear transition to breakdown. In both cases, a region of erratic current transients increase well below the expected breakdown. Although one trace has a transition to a smooth breakdown slope, both tests showed visible breakdown damage, confirming breakdown as per ASTM D3755-14 8.4 [1]. It is evident that in some cases the breakdown rupture leaves some residual resistance in the circuit. Fig. 2 (b) shows plots for faster ramp rate tests at 125 $\mathrm{V} / \mathrm{s}, 300 \mathrm{~V} / \mathrm{s}$, and $500 \mathrm{~V} / \mathrm{s}$. At accelerated rates, it can be especially difficult to identify a transition to an ohmic slope, if the expected ohmic slope is even completely achieved. Faster ramp rates also suffer from a decrease in the accuracy of measured breakdown voltage and loss of information about prebreakdown phenomena [9].

\section{ENHANCED BREAKDOWN CRITERIA}

Taken together, the observations in Sec. II suggest that a single threshold current value-independent of applied voltage and other test conditions-is an insufficient criterion. Further, it is clear that measurements of time and voltage dependent current are useful in establishing a more universal and precise breakdown criterion. A useful criterion for dielectric breakdown must be distinguished from other behaviors, such as:

- PD (transient current spikes)

- Surface flashover (transient current trace like PD in the leakage current, but where the voltage drops such that the point lies on the $R_{l m}$ ohmic curve)

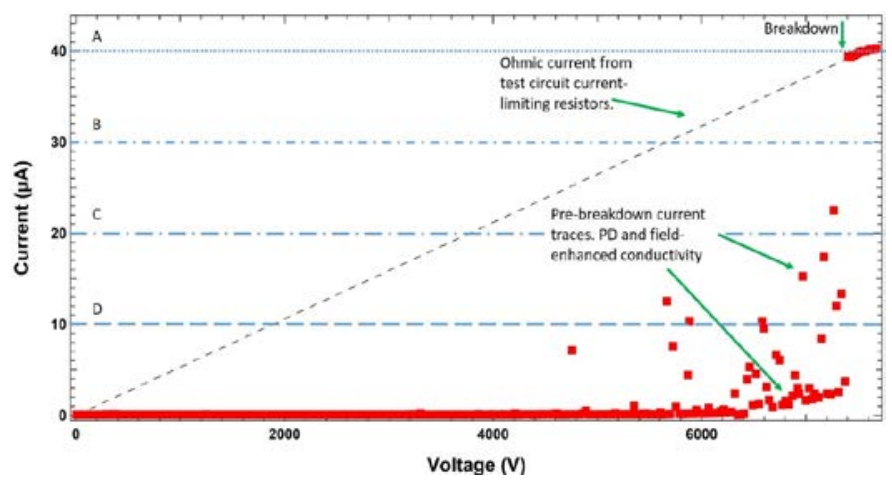

Fig. 1. Current traces from a prototypical voltage step-up test in baked polyimide (PI) under vacuum conditions tested at a $\sim 5 \mathrm{~V} / \mathrm{s}$ ramp rate. At low voltages up to $\sim 3 \mathrm{kV}$, no leakage current is observed. As voltage increases, prebreakdown current traces are observed. In this test, a clear discontinuity in the observed current occurs at breakdown. Supposing a sensing threshold A (40 $\mu \mathrm{A})$, the true breakdown voltage would have been missed. At B $(30 \mu \mathrm{A})$, the true breakdown voltage would be observed for this test. At C and D (20 and 10 $\mu \mathrm{A}$ respectively), partial discharges would erroneously indicate a breakdown voltages below the true value.
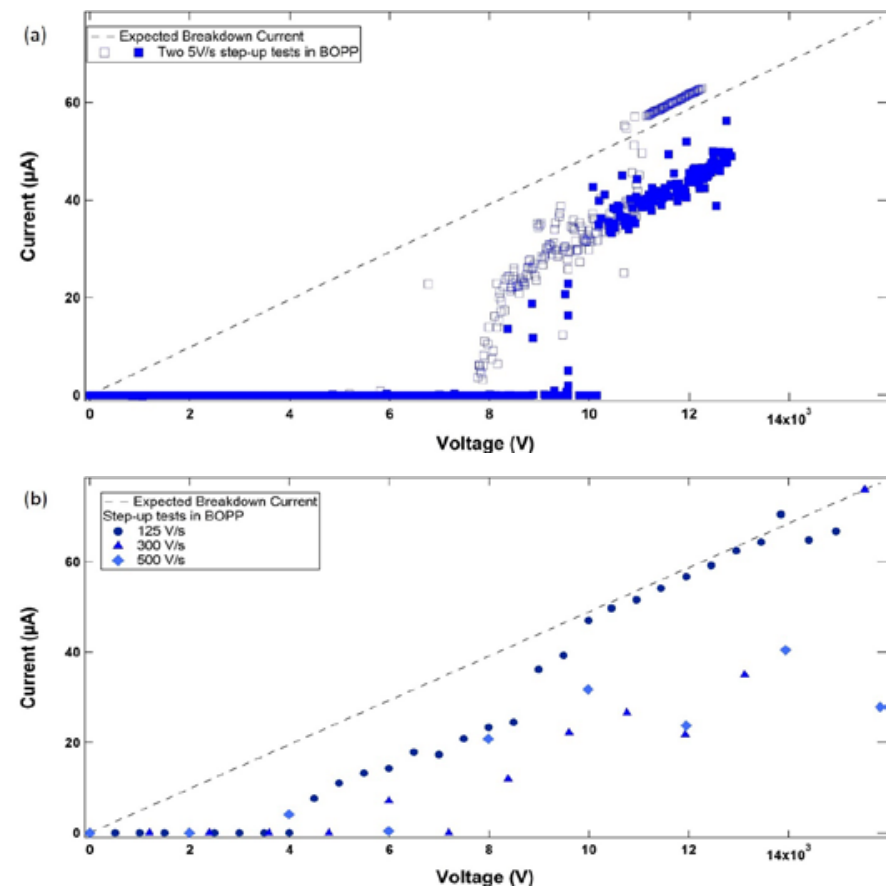

Fig. 2. Current traces from an example voltage step-up tests in biaxiallyoriented polypropylene (BOPP). Dashed lines correspond to Ohms law for the circuit's current limiting resistors. (a) Plot of two step-up tests with $\sim 5 \mathrm{~V} / \mathrm{s}$ ramp rate. In the test with the open markers, an area of erratic traces proceeds the smooth ohmic slope. The test with solid markers does not transition to a smooth slope. (b) Plot of three step up tests at ramp rates of $125 \mathrm{~V} / \mathrm{s}, 300 \mathrm{~V} / \mathrm{s}$, and 500 $\mathrm{V} / \mathrm{s}$. In these cases there is not a clear discontinuity from negligible current to current increasing ohmically with applied voltage.

- Arcing on the external components of the chamber (marked by sudden drops in sustained breakdown currents)

- Field enhanced conductivity (smooth monotonically increasing measureable currents above the noise, but not ohmic)

- Current or voltage meter errors that occasionally happen.

These pre-breakdown phenomena are more easily distinguished using a log current scale, as shown in Fig 3. Further details on 
pre-breakdown current phenomena are given in another paper [4].

A definition of breakdown based on rate of change of leakage current, rather than a simple threshold, automatically filters out transient non-breakdown phenomena. In practice, this requires continuous monitoring of leakage current rather than simply a breaker or fuse. For typical high-resistance dielectric thin film samples, current sensitivity of less than $100 \mathrm{nA}$ is usually required.

Breakdown is defined by the voltage (or field) at which an insulator no longer blocks significant current flow; therefore, even ohmic-like traces below the expected smooth $R_{l m}$ ohmic behavior must be considered breakdowns. NASA spacecraft charging mitigation guidelines state that insulators are considered safe if bulk resistivity is on the order of $10^{12} \Omega \cdot \mathrm{cm}$ or less [5]. Such materials have enough conductivity to bleed away charge fast enough to make breakdown very unlikely. Given our electrode area of $1.98 \mathrm{~cm}^{2}$ and a typical sample thickness of about $25 \mu \mathrm{m}$, this threshold resistivity is equivalent to a sample resistance of $\sim 10^{9} \Omega$ compared to our $R_{l m} \sim 2 \cdot 10^{6} \Omega$ [2]. Thus, such 'safe' materials would have slopes on the order of $\sim 10 \%$ of the $R_{l m}$ ohmic breakdown curve.

Based on the considerations outline above, we propose the following improved operational definition of the electrostatic breakdown voltage for step-up tests:

Breakdown voltage can be defined as the average voltage between the last voltage with current below $10 \%$ of the $R_{l m}$ expected ohmic breakdown curve and the first voltage with current increasing (though not necessarily smoothly) between $10 \%$ and $110 \%$ of the $R_{l m}$ ohmic breakdown curve.

In some test configurations, such as static voltage endurance time (SVET) tests, current versus time (rather than voltage) or some other variable may be of interest. For SVET tests, current transitions from a baseline to a constant value at breakdown. Any intermediate behavior is likely to happen over such small time increments compared to the SVET wait time that they may be absorbed into the uncertainty without much consequence. There is the possibility of one or more points dropping back below the $10 \%$ curve due to external arcing after an apparent breakdown. This is not very frequent and is generally an obvious deviation from a breakdown slope.

Since PD and flashover generally return to baseline current, they will not qualify as breakdown using this definition. Increases in current significantly steeper than the $R_{l m}$ ohmic curve do not make sense in terms of Ohm's law (V=IR) for the circuit; these are likely due to the ammeter response to increasingly rapid pre-arcs—so rapid that return to baseline current may not be observed [4, 10]. Much, if not all, field enhanced conductivity (which is relatively infrequent in MPG tests) will be below the $10 \%$ curve; further, field enhance conductivity is expected to increase supra-linearly with voltage and can therefore be distinguished from breakdown.

Fig. 4 revisits the same example tests from Fig. 2. Regions of current increasing linearly with voltage above the minimum breakdown current (red dashed line) are identified with orange lines. In Fig. 4 (a) a grey line indicates a slope corresponding to

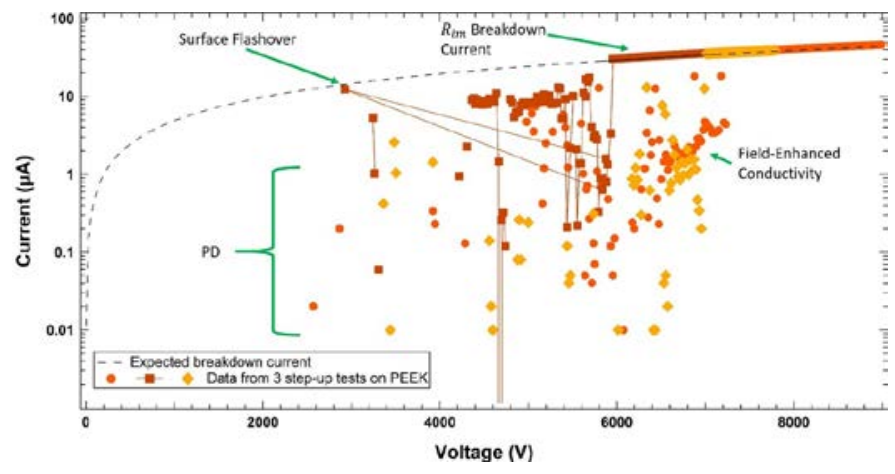

Fig. 3. Current traces from three example voltage step-up tests in polyether ether ketone (PEEK) at $\sim 5 \mathrm{~V} / \mathrm{s}$. The log current scale facilitates the identification of surface flashover, and field-enhanced conductivity. PD is also observed.
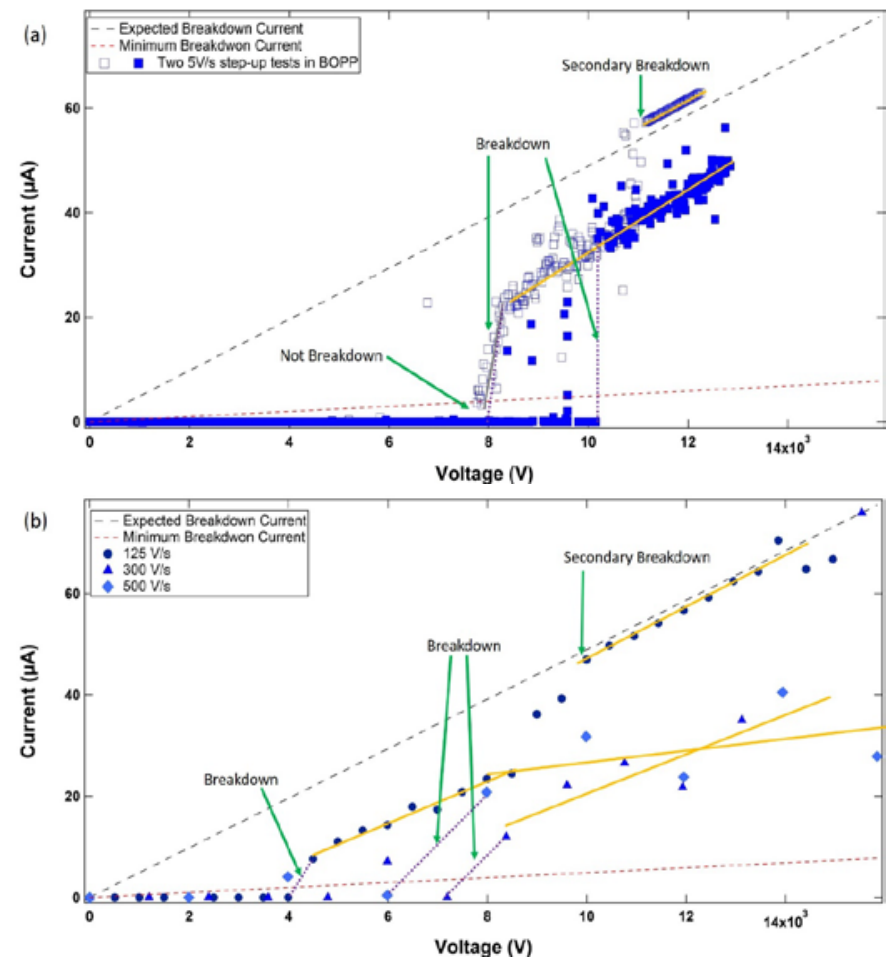

Fig. 4. Data from the same tests in Fig. 2 with breakdowns identified using proposed criteria. Orange lines are linear fits to breakdown current regions. Purple dotted lines connect the last baseline current datum with the first point corresponding to a measurement of breakdown. The breakdown voltage is the average of these two points with its uncertainty dominated by the spread of these two points. In (a) the grey line has slope corresponding to a resistance much less than the current limiting resistors and therefore cannot be identified as breakdown. Secondary breakdowns to the $R_{l m}$ ohmic slope are shown in two cases; however, they are not reported as the primary breakdown.

a resistance much less than $R_{l m}$. Purple dashed lines connect the last sub-breakdown point to the first point of a breakdown region. The average voltage of these two points-the last measurement of a sample not yet broken down and the first measurement corresponding to a broken down sample-is identified as the breakdown voltage. The voltage difference between these two points contributes to the measurement uncertainty but is often small, especially for slow voltage ramp rates. Secondary or even tertiary breakdowns can be observed 
after incomplete breakdowns; however, the initial breakdown is the primary failure. Using this criterion for data with an obvious transition to breakdown, as in Fig. 1, does not change the value of the breakdown voltage.

\section{CONCLUSIONS}

We propose that dielectric breakdown voltage be defined more precisely as the average voltage between the last voltage with current below $10 \%$ of the $R_{l m}$ expected ohmic breakdown curve and the first voltage with current increasing between $10 \%$ and $110 \%$ of the $R_{l m}$ ohmic breakdown curve.

This definition requires continuous, or at least intermittent, monitoring of low-level leakage currents; it is, however, insensitive to false negatives or false positives that may arise from using a breakdown current threshold criterion as outlined in ASTM Standard D3755. The identification of breakdown in fast voltage ramp-rate tests is also improved.

\section{ACKNOWLEDGMENT}

This work was supported by a NASA Space Technology Research Fellowship.

\section{REFERENCES}

[1] Standard Test Method for Dielectric Breakdown Voltage and Dielectric Strength of Solid Electrical Insulating Materials Under Direct-Voltage Stress, document ASTM D3755-14, 2014.

[2] A. Andersen, J. R. Dennison, A. M. Sim, and C. Sim, "Measurements of Endurance Time for Electrostatic Discharge of Spacecraft Materials: A Defect-Driven Dynamic Model," Plasma Science, IEEE Transactions on, vol. 43, pp. 2941-2953, 2015.

[3] A. Andersen, K. Moser, and J. Dennison, "Perspectives on the Distributions of ESD Breakdowns for Spacecraft Charging Applications," IEEE Tran. Plasma Science. 2017, in press.

[4] A. Andersen and J. Dennison, "Highly Accelerated Test Method for Characterizing Likelihood of Breakdown in HVDC Dielectric Materials," IEEE Transactions on Dielectrics and Electrical Insulation, 2017, in press.

[5] Mitigating in space charging effects-a guideline, document NASAHDBK-4002A, 2011.

[6] Avoiding problems caused by spacecraft on-orbit internal charging effects, NASA-HDBK-4002, 1999.

[7] H. B. Garrett and A. C. Whittlesey, Guide to mitigating spacecraft charging effects: John Wiley \& Sons, 2012.

[8] J. Brunson, "Hopping conductivity and charge transport in low density polyethylene," 2010.

[9] K. Moser, A. Andersen, and J. Dennison, "Dependence of Electrostatic Field Strength on Voltage Ramp Rate for Spacecraft Materials," IEEE Tran. Plasma Science. 2017, in press.

[10] A. Andersen and J. Dennison, "Pre-breakdown Arcing and Electrostatic Discharge in Dielectrics under High DC Electric Field Stress," 2014 Annual Report Conference on Electrical Insulation and Dielectric Phenomena, pp. 63-66, 2014. 